\title{
Synthesis of 8-Aza-3-deazaisoguanosine by a Novel Ring Closure of Dinitriles by Sodium Alkoxides*
}

\author{
Marjan Ješelnik, Suzana Jakša, and Jože Kobe**
}

National Institute of Chemistry, Hajdrihova 19, SI-1000 Ljubljana, Slovenia.

RECEIVED MARCH 10, 2003; REVISED JULY 3, 2003; ACCEPTED JULY 11, 2003

\begin{abstract}
Key words This communication describes a convenient, direct ring closure of a novel 4-cyano-5-cyanomesynthesis thyl-1-( $\beta$-D-ribofuranosyl)-1,2,3-triazole under basic conditions into 4-amino-6-alkoxy-1-( $\beta$ 8-aza-3-deazaisoguanosine D-ribofuranosyl)-1,2,3-triazolo[4,5-c]pyridine, which can be easily transformed to 8-aza-3-dering closure of dinitriles azaisoguanosine.
\end{abstract}

\section{INTRODUCTION}

A nucleobase isomer such as isoguanine apparently makes a non-standard base pair between 2'-deoxyisocytidine and 2'-deoxyisoguanosine feasible when incorporated into oligomers. Some levels of functions in this isomeric replacement are retained and in some instances new functions are formed. The results reported by Switzer et al. ${ }^{1}$ have shown that various polymerases handle this non-standard base pair, while Horn et al. ${ }^{2}$ reported on the pairing in the absence of the enzyme. Here we report on a subtle change in the molecular shape by replacing $\mathrm{N}^{3}$-nitrogen with the more liphophilic $\mathrm{C}^{3} \mathrm{H}$ group and $\mathrm{C}^{8} \mathrm{H}$ by $\mathrm{N}^{8}$-nitrogen. Though we do not prevent the Watson-Crick pairing, the ring stacking might be altered. The absence of $\mathrm{N}^{3}$-nitrogen in guanine introduces an incorrectness that could disrupt the minor groove spine of hydration, which means that it should eliminate the presence of any ordered water molecules or metal ions at this sequence position. ${ }^{3}$ Recently, it has been demonstrated that in $\mathrm{dA}-\mathrm{dT}$ sequences, the spine of hydration should be considered an integral part of the helix and that the possible metal ions binding in the minor groove is critical for the structural integrity of duplex DNA. ${ }^{4}$

In the light of these statements, our intention to design and carry out efficient synthesis of 8-aza-3-deazaisoguanosine 2 seemed attractive and significant especially when self-assembled properties of this molecule are considered. Namely, guanosine (G) derivatives self-associate in the presence of metal ions to give hydrogen-bonded G-quartets ${ }^{5}$, while $\mathrm{Cs}^{+}$-selective ionophore, properly protected isoG can also form a hydrogen-bonded pentamer in the cation-templated process. ${ }^{6}$ These covalent assemblies selectively co-ordinate monovalent and divalent cations to give extremely stable complexes. Lack of one potential $\mathrm{H}$-bond per molecule at $\mathrm{N}^{3}$-nitrogen donor may certainly weaken the H-bond pattern though leaving sugar-base H-bonds transmitting stereochemical information intact if the aggregates were formed as in the isoG series. ${ }^{7}$ Therefore, considerable alternations in binding affinity and selectivity are expected when using this potential ionophore. This model can be

* Dedicated to Professor Nenad Trinajstić on the occasion of his $65^{\text {th }}$ birthday.

** Author to whom correspondence should be addressed. (E-mail: joze.kobe@ki.si) 
further extended to its use in the search for structure-activity relationships concerning G-rich oligodeoxynucleotides (ODN) that may form G4-stabilized tetramers on monovalent cations as templates or without. ${ }^{8} \mathrm{G} 4$ or isoG4 containing structures may act as negative regulators of telomerase elongation in vivo due to their ability to inhibit telomerase in vitro ${ }^{9}$ and it was demonstrated that such ODNs are potent inhibitors of HIV-1 integrase. ${ }^{10}$ Seela et al. ${ }^{11}$ have recently established that the isoG quartet is generally more stable than the G-quartet on the first defined tetraplex species; however, structural changes of a heterocyclic base resulted in somewhat lower stability for 7 -deazaisoguanine quartet. ${ }^{11 b}$
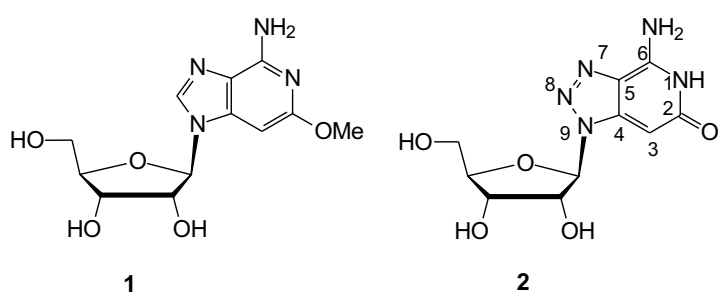

Figure 1. 8-Aza-3-deazaisoguanosine 2 (purine numbering).

Despite remarkable interest in isoguanosine physical and structural nature as well as in its 2-O-methylated congener spongosine ${ }^{12} \mathbf{1}$, there have been no attempts to perform the synthesis of 3-deaza analogues (Figure 1). Strategically, the linear approach to 8-azapurine ring rebuilding and achieving the synthesis of the corresponding modifications relies on the availability of appropriate 5-member heterocyclic nucleoside intermediates suitable for subsequent ring closure (Figure 2). ${ }^{13}$
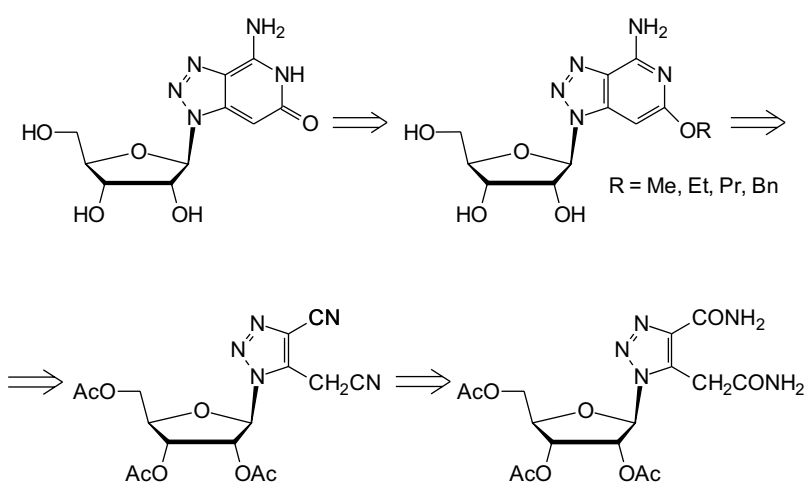

Figure 2. Retrosynthetic scheme.

\section{RESULTS AND DISCUSSION}

We have recently reported on a high-yield procedure for the preparation of various 8-aza-3-deazaguanosine analogues, which enabled us to select one of the two possible routes. ${ }^{13 a}$ One could follow the obvious work-out of the parent ring system of 1-substituted-1,2,3-triazolo- [4,5-c] pyridine by subsequent transformations. More elegant and certainly much more efficient could be a directed cyclization into the target compounds. Apparently, the most convenient way to yield any 3-deazaguanosine analogue by a ring closure, requires the key intermediate 4-ethoxycarbonyl or 5-cyanomethyl-1-(2,3,5-triacetyl- $\beta$ -D-ribofuranosyl)imidazole and/or (1,2,3-triazole)-4-carboxamide $(\mathrm{X}=\mathrm{CH}, \mathrm{N}),{ }^{14}$ namely a mononitrile-methoxycarbonylmethyl function.
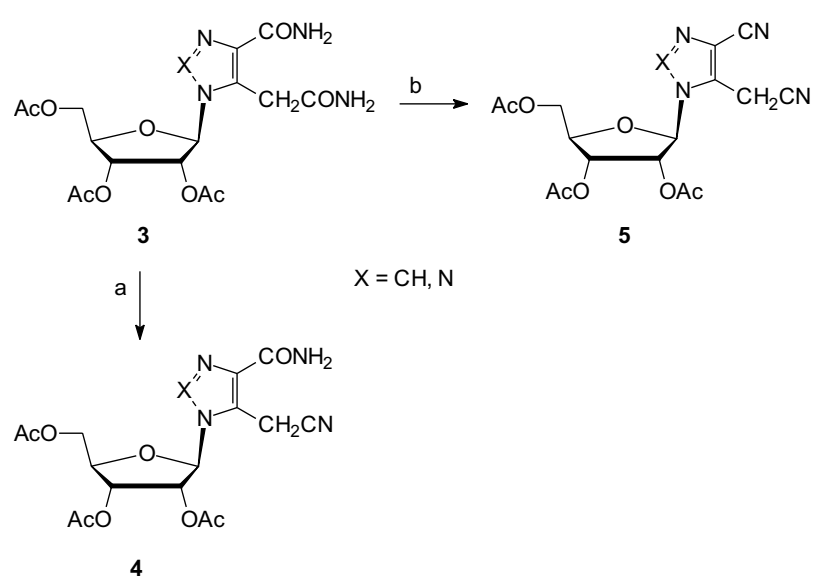

Scheme 1. Synthesis of intermediates 4 and 5: a) 1.1 eq. $\left(\mathrm{CF}_{3} \mathrm{CO}\right)_{2} \mathrm{O}$, pyridine, $\mathrm{THF},-10{ }^{\circ} \mathrm{C}$; b) 2.2 eq. $\left(\mathrm{CF}_{3} \mathrm{CO}\right)_{2} \mathrm{O}$, pyridine, $\mathrm{THF},-10^{\circ} \mathrm{C}$.

The anticipated regioselective pathway requires an aromatic nitrile in the place of a carboxylate function that might reverse the course of the ring closure in methanolic ammonia. ${ }^{15}$ However, it seems that no aromatic carboxamide precursor has ever been detected in the work-out of a controlled selective ammonolysis of 5-methoxycarbonylmethyl-1- $\beta$-D-ribofuranosyl-1,2,3-triazole-4-carboxylates. ${ }^{13}$ On the other hand, the diamide 3 $(\mathrm{X}=\mathrm{N})$ appeared in minor quantities in a reaction at $0{ }^{\circ} \mathrm{C}$ and, by raising the temperature up to ambient conditions, a $91 \%$ yield of $\mathbf{3}(\mathrm{X}=\mathrm{N})$ was obtained. 3 was then readily converted to dinitrile $5(X=N)$ in dry THF at $-10{ }^{\circ} \mathrm{C}$ to $-2{ }^{\circ} \mathrm{C}$ in the presence of pyridine (Eq. 4) with careful addition of 2.2 eq. of trifluoroacetic acid anhydride in THF (Scheme 1).

Intramolecular cyclizations between a cyanomethyl group and an adjacent cyano group located on benzene, ${ }^{16}$ pyridine, ${ }^{17}$ or imidazole ${ }^{18}$ ring to form isoquinolines, pyrido $[c]$ pyridines and imidazo[4,5-c]pyridines under acidcatalyzed conditions have been reported. In addition, use of hydrogen sulfide and triethylamine at room temperature took the same cyclization course. ${ }^{14 a}$ The same authors described also the mild ring closure of $5(\mathrm{X}=\mathrm{CH})$ to yield 2,6-diamino-3-deazaguanosine in methanolic ammonia, which points to a different acting mechanism under base-catalyzed conditions. 
A catalytic amount of sodium methoxide was added to a stirred solution of $\mathbf{5}(\mathrm{X}=\mathrm{N})$. After stirring (30 min) and subsequent adjustment of $\mathrm{pH}(6.5-7)$, a new product tentatively $\mathbf{6}$ was isolated from the solution (Scheme 2).

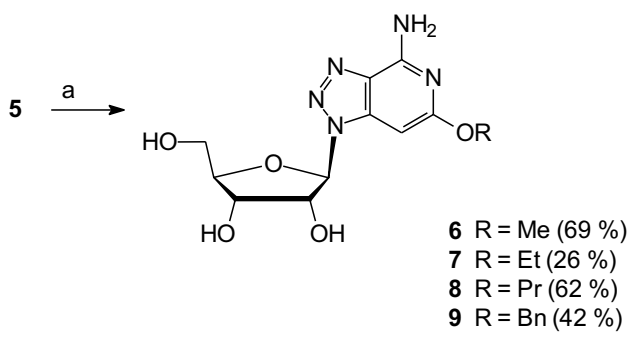

Scheme 2. Ring closure reaction of dinitrile by sodium alkoxides: a) $\mathrm{NaOR}$ in $\mathrm{ROH}$.

In the ${ }^{1} \mathrm{H}$ NMR spectrum in DMSO- $d_{6}$, a new aromatic proton appeared at $\delta 6.30$, two exchangeable protons at $\delta 7.25$, apparently belonging to the amino group, and a sharp peak at $\delta 3.79$, which was assigned to the newly formed $\mathrm{OCH}_{3}$ substituent. Hitherto no NMR data was available for comparison, yet the readily available $\mathbf{1 0}$ in our hands presumably implies an independent synthetic approach in classical chemical transformations of this interesting ring system, thus providing us with an unambiguous structural proof (Scheme 3).
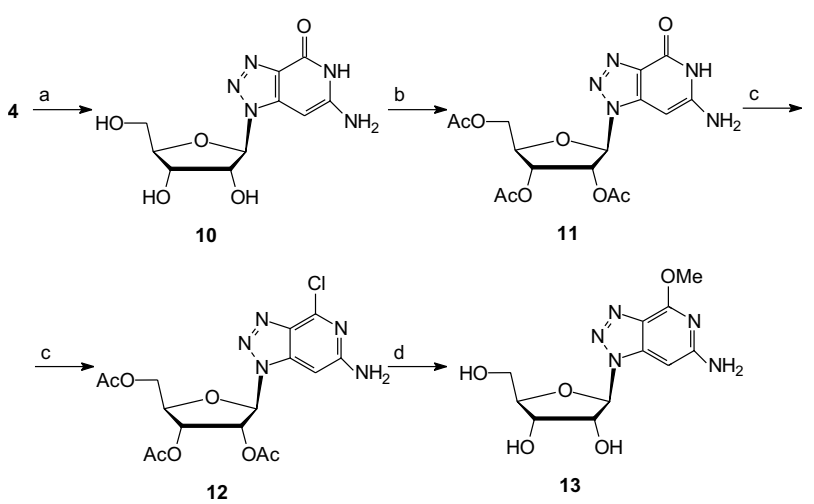

Scheme 3. Formation of isomeric 13 from 4: a) $1 \mathrm{M} \mathrm{NEt}_{3}$ in $80 \% \mathrm{MeOH}$; b) $\mathrm{Ac}_{2} \mathrm{O}$, pyridine, $\mathrm{DMF}$; c) $\mathrm{POCl}_{3}$, collidine, $\mathrm{NEt}_{4} \mathrm{Cl}$, $\mathrm{MeCN}$; d) $\mathrm{NaOMe}$ in $\mathrm{MeOH}$.

Acetylation of $\mathbf{1 0}$ afforded the suitable intermediate 11, which was further chlorinated with $\mathrm{POCl}_{3}$ in acetonitrile in the presence of collidine and $\mathrm{NEt}_{4} \mathrm{Cl}$ in $70 \%$ yield. The leaving group in $\mathbf{1 2}$ turned to be, contrary to that in the related ring system, ${ }^{19}$ quite a versatile precursor. First, methanololysis with sodium methoxide in methanol in a pressure bottle at $120{ }^{\circ} \mathrm{C}$, adjusting the $\mathrm{pH}$ to 6.6-7.0, and final purification by column chromatography has furnished 13 (Scheme 3). ${ }^{1} \mathrm{H}$ NMR data revealed distinct numbers from $6\left\{{ }^{1} \mathrm{H}\right.$ NMR $\delta / \mathrm{ppm}$ (DMSO- $\left.d_{6}\right) 6.07$ (H-3), $6.18\left(\mathrm{NH}_{2}\right)$ and $\left.4.00\left(\mathrm{OCH}_{3}\right)\right\}$. MS (FAB), $\mathrm{m} / \mathrm{z}=$ $298\left(\mathrm{M}^{+1}\right)$, produced no notable change but a protonated base $(m / z=166)$ in CID-Mike spectra of $\mathrm{BH}^{+}\left(\right.$or $\left.\mathrm{BH}^{2+}\right)$. Careful hydrogenation of $\mathbf{1 2}$ afforded $\mathbf{1 4}$ (Scheme 5) $\left\{{ }^{1} \mathrm{H} \text { NMR } \delta / \mathrm{ppm} \text { (DMSO-d }\right)_{6} 8.87$ (H-6), 6.79 (H-3, $\left.\left.J_{3,6}=0.98 \mathrm{~Hz}\right)\right\}$, which had not been previously reported. 3,6-Diamino compound 15 was obtained $\left\{{ }^{1} \mathrm{H}\right.$ NMR $\delta /$ ppm (DMSO- $\left.d_{6}\right) 5.72(\mathrm{H}-3), 7.62,7.24\left(4 \mathrm{H}, \mathrm{bs}, \mathrm{NH}_{2}\right)$, $\left.5.87\left(1 \mathrm{H}, \mathrm{d}, \mathrm{H}-1^{\prime}\right)\right\}$ after heating 12 in liquid ammonia for 16 hours. This structural correlation has definitely proven that a novel synthetic method to 8-aza-3deazaspongosine analogues is available by the sodium methoxide catalyst procedure. To make this statement more general, several sodium alkoxides were employed. The results are depicted in Scheme 2. The use of different sodium alkoxides ${ }^{17}$ directed the cyclization of aromatic (heterocyclic) nitriles with an adjacent cyanomethyl group, e.g. the nitrogen of the aliphatic cyano group becoming the ring nitrogen of the pyridine moiety, in the »reverse « way to the acid-catalyzed intramolecular cyclizations. ${ }^{15 a}$ This is the first successful example in nucleoside chemistry and the methodology is certainly of high potential for the design of 2-alkoxy-8-aza-3-deaza-6-aminopurines aimed as potential coronary vasodilators. ${ }^{20}$ The procedure corroborates some of the earlier attempts ${ }^{21}$ relating to this type of ring closures. We can conclude that catalytic amounts of the appropriate alkoxide are sufficient to generate the desired product. However, more nucleophile (2, 3, 4 or 5 equivalents) used in the reaction afforded a $20 \%$ yield of the isomeric product 13. In that case, addition of excess methoxide to the aromatic nitrile might generate an imminium ion, which then undergoes cyclization to the cyanomethyl group. ${ }^{21 a}$ To take advantage of alkoxides bearing removable alkyl groups, 8-aza-3-deazaisoguanosine $\mathbf{2}$ was obtained by standard deprotection of the benzylic group.

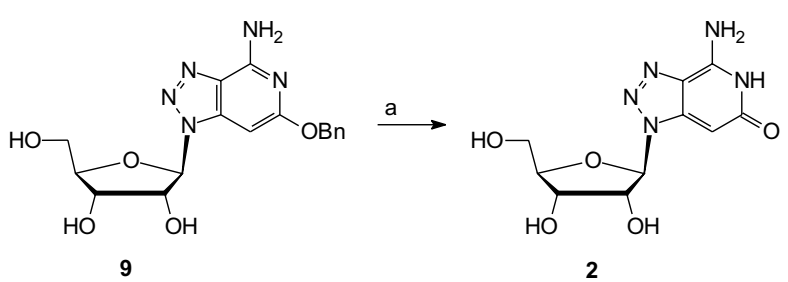

Scheme 4. Removal of the benzyl group from 9: a) $\mathrm{H}_{2} / 5 \%$ $\mathrm{Pd} / \mathrm{C}, 80 \% \mathrm{EtOH}$.

Finally, intermediate $\mathbf{3}$ was recognized as the anticipated precursor for providing the target nucleoside 2 . Our synthetic methodology was initially designed to carry on the desired sugar configuration through subsequent steps.

The use of 2',3'-protected $\mathbf{6}$ allowed the application of the Imbachs rule ${ }^{22}\left(\Delta \delta \mathrm{Me}\right.$ in $\left.\mathrm{C}\left(\mathrm{CH}_{3}\right)_{2}=17.5 \mathrm{~Hz}\right)$ for 
the assignment of $\beta$-configuration of $\mathbf{6}$ and $\mathbf{2}$, which was readily obtained by catalytic hydrogenation of $\mathbf{9}$ in $80 \%$ ethanol and $5 \% \mathrm{Pd} / \mathrm{C}$. $\left\{{ }^{1} \mathrm{H}\right.$ NMR $\left(\mathrm{DMSO}-d_{6}\right) \delta / \mathrm{ppm}$ $10.4\left(1 \mathrm{H}\right.$, bs, NH), $7.29\left(2 \mathrm{H}\right.$, bs, $\left.\mathrm{NH}_{2}\right), 5.88(1 \mathrm{H}, \mathrm{d}$, $\left.\left.\mathrm{H}-1^{\prime}\right)\right\}$. In addition, in the attempted cleavage of the ether bond in $\mathbf{6}$ in methanol, saturated with gaseous $\mathrm{HCl}$ at ambient temperature, depurination was observed, providing 4-amino-6-methoxy-1H-1,2,3-triazolo[4,5-c]pyridine.

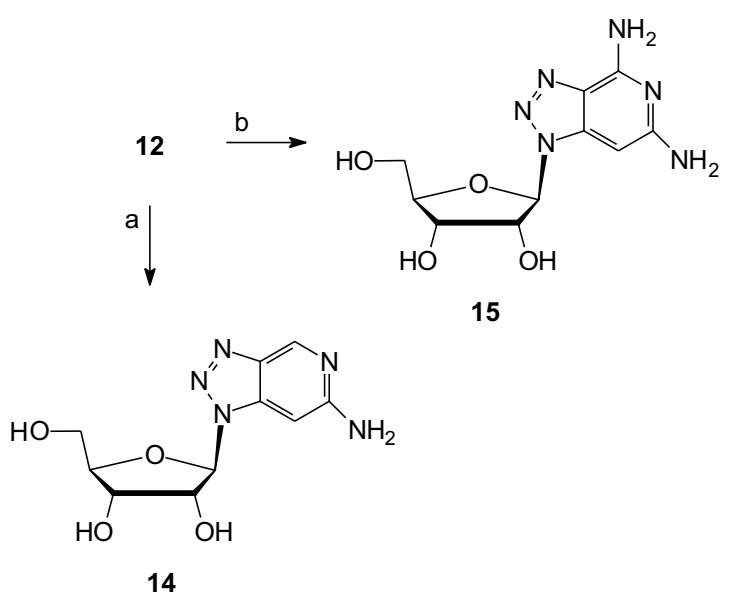

Scheme 5. Synthesis of new derivatives 14 and 15: a) $\mathrm{H}_{2} / 5 \%$ $\mathrm{Pd} / \mathrm{C}, \mathrm{E \dagger OH} ; \mathrm{b}) \mathrm{NH}_{3}(I), 110^{\circ} \mathrm{C}$.

\section{EXPERIMENTAL}

\section{General}

${ }^{1} \mathrm{H}$ and ${ }^{13} \mathrm{C}$ spectra were obtained in DMSO- $d_{6}$, and chemical shifts are reported on the $\delta$-scale from TMS as an internal standard. Melting points were obtained on a Kofler micro stage and are uncorrected. IR spectra were obtained on a Bio-Rad FTS 15/80 spectrometer and mass spectra on an Avtospec Q spectrometer. All compounds gave satisfactory elemental analyses or HRMS.

2-[1-(2,3,5-tri-O-Acetyl- $\beta$-D-ribofuranosyl)-4-carboxamide-1,2,3-triazolo-5-yl]acetamide (3)

5-Methoxycarbonylmethyl-1-( $\beta$-D-ribofuranosyl)-1,2,3-triazole-4-carboxylate ${ }^{13 \mathrm{~g}}(42.0 \mathrm{~g}, 127 \mathrm{mmol})$ was dissolved in saturated $\mathrm{NH}_{3}\left(0{ }^{\circ} \mathrm{C}\right)$ in $\mathrm{MeOH}(700 \mathrm{ml})$, stirred at room temperature for $24 \mathrm{~h}$ and the solvent evaporated to furnish $39.9 \mathrm{~g}(95 \%)$ of red oil which was acetylated without further purification.

IR (film) $v_{\max } / \mathrm{cm}^{-1}: 3464,3131,1599,1285,691,540 ;$ ${ }^{1} \mathrm{H}$ NMR $\left(\mathrm{D}_{2} \mathrm{O}\right) \delta / \mathrm{ppm}: 5.83\left(1 \mathrm{H}, \mathrm{d}, \mathrm{H}-1^{\prime}, J_{1^{\prime} 2^{\prime}}=2.7 \mathrm{~Hz}\right)$, $4.71\left(1 \mathrm{H}, \mathrm{dd}, \mathrm{H}-2^{\prime}, J_{2^{\prime} 3^{\prime}}=4.6 \mathrm{~Hz}\right), 4.28\left(1 \mathrm{H}, \mathrm{m}, \mathrm{H}-3^{\prime}\right), 3.97$ $\left(1 \mathrm{H}, \mathrm{m}, \mathrm{H}-4^{\prime}\right), 3.94,4.17$ (2H, 2d, $\left.\mathrm{CH}_{2} \mathrm{CO}\right), 3.55(1 \mathrm{H}, \mathrm{dd}$, H-5'a), 3.36 (1H, dd, H-5'b); MS (FAB) m/z: $302\left(\mathrm{MH}^{+}\right)$.

The intermediate diamide (38.66 $\mathrm{g}, 128.3 \mathrm{mmol})$ was dissolved in dry acetonitrile (500 ml), DMAP (1.18 g, 9.6 $\mathrm{mmol})$ in pyridine $(70.7 \mathrm{ml})$ was added and acetic anhydride $(43.7 \mathrm{ml}, 462 \mathrm{mmol})$ was added dropwise under vigorous stirring. The reaction was carefully followed by TLC
$\left(\mathrm{CH}_{2} \mathrm{Cl}_{2} / \mathrm{CH}_{3} \mathrm{OH}=4: 1\right)$. After o few hours of stirring, the mixture was evaporated and the resulting residue was coevaporated with water (twice). The oily residue was then dried, applied onto a silica gel column $(250 \mathrm{~g})$ and eluted with a gradient solvent mixture of acetone in EtOAc (25$40 \%)$ to yield the white crystalline solid 3 (26.3 g, $48 \%$, m.p. $\left.=173-174{ }^{\circ} \mathrm{C}\right)$; IR (KBr) $v_{\max } / \mathrm{cm}^{-1}: 3449,1751,167$, 1242, 1061, 617; ${ }^{1} \mathrm{H}$ NMR (DMSO- $d_{6}$ ) $\delta / \mathrm{ppm}: 7.64,7.36$, 7.07, $6.64(4 \mathrm{H}, 4$ x br. s, 2 x NH 2$), 6.39\left(1 \mathrm{H}, \mathrm{d}, \mathrm{H}-1^{\prime}, J_{1^{\prime} 2^{\prime}}=\right.$ $2.5 \mathrm{~Hz}), 6.15\left(1 \mathrm{H}, \mathrm{dd}, \mathrm{H}-2^{\prime}\right), 5.79\left(1 \mathrm{H}, \mathrm{dd}, \mathrm{H}-3^{\prime}\right), 4.53(1 \mathrm{H}$, m, H-4'), 4.41 (1H, m, H-5'a), 4.15 (1H, m, H-5'b), 3.98 (2H, $\left.\mathrm{CH}_{2} \mathrm{CO}\right), 2.12,2.08,1.92\left(3 \times \mathrm{CH}_{3} \mathrm{CO}\right) ;{ }^{13} \mathrm{C} \mathrm{NMR}$ (DMSO-d $)_{6} \delta / \mathrm{ppm}: 170.11,169.65,169.35\left(3 \times \mathrm{COCH}_{3}\right)$, 168.80, 162.57 (2 x CONH$\left.{ }_{2}\right), 139.22$ (C-4), 135.73 (C-5), 88.10 (C-1'), 79.97 (C-4'), 73.61 (C-2'), 70.26 (C-3'), 62.55 (C-5'), $29.13\left(\underline{\mathrm{CH}}_{2} \mathrm{CO}\right), 20.39,20.36$ (3 x $\left.\mathrm{COCH}_{3}\right)$; MS (FAB) $m / z: 428\left(\mathrm{MH}^{+}, 62 \%\right)$.

\section{4-Cyano-5-cyanomethyl-1-(2,3,5-tri-O-acetyl- $\beta$-D-ribo-} furanosyl)-1,2,3-triazole (5)

Diamide 3 (2.7 g, $6.3 \mathrm{mmol}$, dried at $60{ }^{\circ} \mathrm{C}, 4$ days over $\left.\mathrm{P}_{2} \mathrm{O}_{5}\right)$ was dissolved in dry THF $\left(40 \mathrm{ml}\right.$, dried with $\mathrm{LiAlH}_{4}$ and distilled before use) and pyridine ( $2.13 \mathrm{ml}, 26.5 \mathrm{mmol})$ was added. The reaction mixture was cooled to -10 to $0{ }^{\circ} \mathrm{C}$ and then trifluoroacetic anhydride (TFA, $1.9 \mathrm{ml}, 13.3 \mathrm{mmol}$ ) in THF $(5 \mathrm{ml})$ was added dropwise. Additional stirring at 0 ${ }^{\circ} \mathrm{C}(30 \mathrm{~min})$ and two hours at room temperature were applied before evaporation of the mixture to dryness and its dissolving in dichloromethane $(40 \mathrm{ml})$. Water $(40 \mathrm{ml})$ was added and the organic phase was separated and dried over $\mathrm{Na}_{2} \mathrm{SO}_{4}$, and filtered to furnish dark oil, which was further processed by column chromatography on silica gel (100 g) with hexane / EtOAc $=2: 3$. Yellow crystals were separated after evaporation $(2.23 \mathrm{~g}, 90 \%)$ and recrystallized from EtOAc (m.p. $\left.=98-99.5{ }^{\circ} \mathrm{C}\right)$; IR $(\mathrm{KBr}) v_{\max } / \mathrm{cm}^{-1}$ : 2940, 2250, 1373, 1230, 1062, 921; MS (CI, NH N $_{3} / z: 409$ $\left(\mathrm{MNH}_{4}{ }^{+}, 29 \%\right) ;{ }^{1} \mathrm{H}$ NMR (DMSO-d $\left.d_{6}\right) \delta / \mathrm{ppm}: 1.85,2.08$, $2.15\left(9 \mathrm{H}, 3 \mathrm{~s}, \mathrm{CH}_{3} \mathrm{CO}\right), 4.16,4.69\left(2 \mathrm{H}, 2 \mathrm{~d}, \mathrm{CH}_{2} \mathrm{CN}\right), 4.25$ (1H, dd, H-5b'), 4.07(11H, m, H-5a'), $6.50\left(1 \mathrm{H}, \mathrm{d}, \mathrm{H}-1^{\prime}\right)$, $6.08\left(1 \mathrm{H}, \mathrm{dd}, \mathrm{H}-2^{\prime}\right), 5.60\left(1 \mathrm{H}, \mathrm{m}, 1 \mathrm{H}, \mathrm{H}-3{ }^{\prime}\right) ;{ }^{13} \mathrm{C} \mathrm{NMR}$ $\delta / \mathrm{ppm}: 169.70,169.37,169.13\left(3 \times \mathrm{COCH}_{3}\right), 136.46$, 120.50 ( 2 x CN), 114.16 (C-4), 110.72 (C-5), 88.67 (C-1'), 80.52 (C-4'), 73.19 (C-2'), 69.77 (C-3'), 62.05 (C-5'), 20.0, 25.0, 20.14 (3 x COCH 3$), 12.70\left(\underline{\mathrm{CH}}_{2} \mathrm{CN}\right)$;

Anal. Calcd. for $\mathrm{C}_{16} \mathrm{H}_{17} \mathrm{~N}_{5} \mathrm{O}_{7}: \mathrm{C} 49.11, \mathrm{H} 4.38, \mathrm{~N}$ $17.90 \%$; found: C 49.13, H 4.38, N $17.73 \%$.

General Procedure for 4-Amino-6-methoxy-1-( $\beta$-D-ribofuranosyl)triazolo[4,5-c]pyridine

Dinitrile 5 (100 mg, $0.26 \mathrm{mmol}$ ) was dissolved in solution of pulverized sodium in an appropriate alcohol $(10 \mathrm{ml}, 0.06$ $\mathrm{M})$ and stirred at room temperature for $30 \mathrm{~min}$. Amberlit IRC-50 (100 mg) was added and stirring was continued to adjust $\mathrm{pH}$ to $6.5-7.0$. The resin was filtered off, washed twice with hot water $(5 \mathrm{ml})$ and the filtrate was evaporated. The solid was collected and crystallized from isopropanol / water $=4: 1$. 


\section{4-Amino-6-methoxy-1-( $\beta$-D-ribofuranosyl)-1,2,3-triazolo- [4,5-c]pyridine (6)}

Yield: $69 \%$ (white powder) m.p. $=190.5-191.5{ }^{\circ} \mathrm{C}$; IR (KBr) $v_{\max } / \mathrm{cm}^{-1}: 3456,3365,1640,1614,1497,1251$, 1212, 1125, 1043; ${ }^{1} \mathrm{H}$ NMR (DMSO- $\left.d_{6}\right) \delta / \mathrm{ppm}: 7.25(2 \mathrm{H}$, $\left.\mathrm{s}, \mathrm{NH}_{2}\right), 6.30(1 \mathrm{H}, \mathrm{s}, \mathrm{H}-7), 6.03\left(1 \mathrm{H}, \mathrm{d}, \mathrm{H}-1^{\prime}, J_{1^{\prime} 2^{\prime}}=5.4 \mathrm{~Hz}\right)$, $4.62\left(1 \mathrm{H}, \mathrm{m}, \mathrm{H}-2^{\prime}\right), 4.20$ (1H, m, H-3'), 3.98 (1H, m, H-4'), 3.79 (s, 3H, OMe), 3.59-3.49 (2H, m, H-5'a,b); ${ }^{13} \mathrm{C}$ NMR (DMSO-d $\left.d_{6}\right) \delta / \mathrm{ppm}: 163.5$ (C-6), 150.9 (C-4), 141.4 (C-C7a), 120.9 (C-3a), 91.0 (C-1'), 86.3 (C-4'), 75.3 (C-7), 73.3 (C-2'), 71.0 (C-C-3'), 62.2 (C-5'), 54.9 (C-OMe); MS (EI) $m / z: 297\left(\mathrm{M}^{+}, 33 \%\right)$.

Anal. Calcd. for $\mathrm{C}_{11} \mathrm{H}_{15} \mathrm{~N}_{5} \mathrm{O}_{5}$ : C 44.46, H 4.97, N 23.35 $\%$; found: C 44.50, H 4.92, N $23.35 \%$.

\section{4-Amino-6-ethoxy-1-( $\beta$-D-ribofuranosyl)-1,2,3-triazolo- [4,5-c]pyridine (7)}

Yield: $26 \%$ (white powder); m.p. $=191-192.5^{\circ} \mathrm{C}$; IR $(\mathrm{KBr})$ $v_{\max } / \mathrm{cm}^{-1}$ : 3334, 3393, 3225, 1654, 1612,1595, 1209, 1128, 1051, 963; ${ }^{1} \mathrm{H}$ NMR (DMSO- $\left.d_{6}\right) \delta /$ ppm: $7.22\left(2 \mathrm{H}, \mathrm{s}, \mathrm{NH}_{2}\right)$, $6.27\left(1 \mathrm{H}, \mathrm{d}, \mathrm{H}-1^{\prime}, J_{1^{\prime} 2^{\prime}}=5.4 \mathrm{~Hz}\right), 4.62\left(1 \mathrm{H}, \mathrm{m}, \mathrm{H}-2^{\prime}\right), 4.20$ $\left(3 \mathrm{H}, \mathrm{m}, \mathrm{H}-3^{\prime}\right.$ and $\left.\mathrm{OCH}_{2} \mathrm{CH}_{3}\right), 3.98(1 \mathrm{H}, \mathrm{m}, \mathrm{H}-4), 3.55(2 \mathrm{H}$, m, H-5'a,b), $1.29\left(3 \mathrm{H}, \mathrm{t}, \mathrm{OCH}_{2} \mathrm{CH}_{3}, J=7 \mathrm{~Hz}\right) ;{ }^{13} \mathrm{C} \mathrm{NMR}$ (DMSO- $d_{6}$ ) $\delta /$ ppm: 162.2 (C-6), 150.5 (C-4), 140.7 (C-7a), 128.2 (C-3a), 90.5 (C-1'), 85.8 (C-4'), 75.1 (C-7), 72.7 (C-2'), 70.5 (C-3'), 61.9 (C-5'), $61.8\left(\mathrm{CH}_{3} \underline{\mathrm{CH}}_{2} \mathrm{O}\right), 14.8\left(\mathrm{CH}_{3} \mathrm{CH}_{2} \mathrm{O}\right)$; MS (FAB) $m / z: 312\left(\mathrm{MH}^{+}\right)$.

Anal. Calcd. for $\mathrm{C}_{12} \mathrm{H}_{17} \mathrm{~N}_{5} \mathrm{O}_{5}$ : C 46.30, H 5.50, N 22.50 $\%$; found: C 46.33, H 5.95, N $22.30 \%$.

\section{4-Amino-6-propoxy-1-( $\beta$-D-ribofuranosyl)-1,2,3-triazolo-} [4,5-c]pyridine $(8)$

Yield: $62 \%$ (white powder); m.p. $=184-185.5{ }^{\circ} \mathrm{C}$; IR $v_{\max } / \mathrm{cm}^{-1}:$ 3405, 3332, 3222, 1653, 1609, 1496, 1204, $1047 ;{ }^{1} \mathrm{H}$ NMR (DMSO- $\left.d_{6}\right) \delta / \mathrm{ppm}: 7.21\left(2 \mathrm{H}, \mathrm{s}, \mathrm{NH}_{2}\right), 6.28$ $(1 \mathrm{H}, \mathrm{s}, \mathrm{H}-7), 6.05\left(1 \mathrm{H}, \mathrm{d}, \mathrm{H}-1^{\prime}, J_{1^{\prime} 2^{\prime}}=5.6 \mathrm{~Hz}\right), 4.64(1 \mathrm{H}, \mathrm{m}$, H-2'), $4.22\left(1 \mathrm{H}, \mathrm{m}, \mathrm{H}-3^{\prime}\right), 4.11\left(2 \mathrm{H}, \mathrm{t}, \mathrm{OCH}_{2} \mathrm{CH}_{2} \mathrm{CH}_{3}, J=\right.$ $6.6 \mathrm{~Hz}), 3.99$ (1H, m, H-4'), 3.56 (2H, m, H-5'a,b), 1.70 (2H, m, $\left.\mathrm{OCH}_{2} \mathrm{CH}_{2} \mathrm{CH}_{3}\right), 0.95\left(3 \mathrm{H}, \mathrm{t}, \mathrm{OCH}_{2} \mathrm{CH}_{2} \mathrm{CH}_{3}, J=7.3 \mathrm{~Hz}\right)$; ${ }^{13} \mathrm{C}$ NMR (DMSO- $d_{6}$ ) $\delta /$ ppm: 162.4 (C-6), 150.5 (C-4), 140.7 (C-7a), 128.2 (C-3a), 90.5 (C-1'), 85.8 (C-4'), 75.0 (C-7), 72.7 (C-2'), $70.5\left(\mathrm{C}-3\right.$ '), $68.8\left(\mathrm{OCH}_{2} \mathrm{CH}_{2} \mathrm{CH}_{3}\right), 61.8$ $\left(\mathrm{OCH}_{2} \mathrm{CH}_{2} \mathrm{CH}_{3}\right), 10.5\left(\mathrm{OCH}_{2} \mathrm{CH}_{2} \mathrm{CH}_{3}\right) ; \mathrm{MS}$ (EI) m/z: 325 $\left(\mathrm{M}^{+}, 22 \%\right)$; HRMS Calcd. for $\mathrm{C}_{13} \mathrm{H}_{19} \mathrm{~N}_{5} \mathrm{O}_{5}$ : 325.139720; found: 325.138619 .

\section{4-Amino-6-benzyloxy-1-( $\beta$-D-ribofuranosyl)-1,2,3-triazolo- [4,5-c]pyridine (9)}

Yield: $42 \%$ (white powder); m.p. $=166-167.5{ }^{\circ} \mathrm{C}$; IR $v_{\max } / \mathrm{cm}^{-1}: 3476,3340,1643,1611,1492,1436,1197$, 1100, 1054; ${ }^{1} \mathrm{H}$ NMR (DMSO-d $\left.)_{6}\right) \delta / \mathrm{ppm}: 7.40(5 \mathrm{H}, \mathrm{m}, \mathrm{Ph})$, $7.31\left(2 \mathrm{H}, \mathrm{s}, \mathrm{NH}_{2}\right), 6.36(1 \mathrm{H}, \mathrm{s}, \mathrm{H}-7), 6.05\left(1 \mathrm{H}, \mathrm{d}, \mathrm{H}-1^{\prime}, J_{1^{\prime} 2^{\prime}}\right.$ = $6.6 \mathrm{~Hz}), 5.30\left(2 \mathrm{H}, \mathrm{s}, \underline{\mathrm{CH}}_{2} \mathrm{Ph}\right), 4.63\left(1 \mathrm{H}, \mathrm{m}, \mathrm{H}-2^{\prime}\right), 4.21$ (1H, m, H-3'), 3.99 (1H, m, H-4'), 3.56 (2H, m, H-5'a,b); ${ }^{13} \mathrm{C}$ NMR (DMSO- $\left.d_{6}\right) \delta / \mathrm{ppm}$ : $161.8(\mathrm{C}-6), 150.5$ (C-4), 140.8 (C-3a), 137.6 (C-7a), 128.4, 128.3, 128.0, 127.9, 127.3 (Ph), 90.5 (C-1'), 85.7 (C-4'), 75.6 (C-7), 72.7 (C-2'),
70.5 (C-3'), $67.5\left(\mathrm{CH}_{2} \mathrm{Ph}\right), 61.8$ (C-5'); MS (EI) m/z: 373 $\left(\mathrm{M}^{+}, 13 \%\right)$; HRMS Calcd. for $\mathrm{C}_{17} \mathrm{H}_{19} \mathrm{~N}_{5} \mathrm{O}_{5}$ : 373.139520; found: 373.138619 .

6-Amino-1-(2,3,5-tri-O-acetyl- $\beta$-D-ribofuranosyl)-1,2,3triazolo[4,5-c]pyridine-4(5H)-on (11)

A solution of 6-amino-1-( $\beta$-D-ribofuranosyl)triazolo[4,5-c]pyridine-4(5H)-on, (1.34 g, $4.7 \mathrm{mmol}$; dried for 2 days over $\mathrm{P}_{2} \mathrm{O}_{5}$ at $\left.110{ }^{\circ} \mathrm{C}\right)$ in dried DMF $(40 \mathrm{ml})$ was treated with pyridine $(1.5 \mathrm{ml}, 18.8 \mathrm{mmol})$ and acetic anhydride $(2.6 \mathrm{ml}$, $18.8 \mathrm{mmol}$ ), which was slowly added under vigorous stirring. The resulting mixture was then heated under reflux for 5 hours and evaporated. The sticky residue was dissolved in isopropanol $(25 \mathrm{ml})$ and refluxed for $15 \mathrm{~min}$, and allowed to cool $\left(-20{ }^{\circ} \mathrm{C}\right)$. The solid was separated overnight to yield $11\left(1.32 \mathrm{~g}, 78 \%\right.$, m.p. $\left.=217-220{ }^{\circ} \mathrm{C}\right)$. IR $(\mathrm{KBr}) v_{\max } / \mathrm{cm}^{-1}$ : 3464, 3343, 3185, 1747, 1683, 1636, 1432, 1373, 1235, 1044, 788; ${ }^{1} \mathrm{H}$ NMR $\left(\mathrm{CDCl}_{3}\right) \delta / \mathrm{ppm}: 10.76$ (1H, br.s, NH), $6.31\left(1 \mathrm{H}, \mathrm{d}, \mathrm{H}-1^{\prime}\right), 6.19$ (2H, br.s, $\left.\mathrm{NH}_{2}\right), 5.95(1 \mathrm{H}, \mathrm{dd}, \mathrm{H}-2$ ', $\left.J_{1^{\prime} 2^{\prime}}=2.7 \mathrm{~Hz}\right), 5.61\left(1 \mathrm{H}, \mathrm{dd}, \mathrm{H}-3^{\prime}\right), 5.49(1 \mathrm{H}, \mathrm{s}, \mathrm{H}-7), 4.45$ $\left(1 \mathrm{H}, \mathrm{m}, \mathrm{H}-4{ }^{\prime}\right), 4.34(1 \mathrm{H}, \mathrm{m}, 1 \mathrm{H}, \mathrm{H}-5 \mathrm{\prime}), 4.05(1 \mathrm{H}, \mathrm{dd}$, $\mathrm{H}-5$ 'b), 2.12, 2.11, 1.91 (3 x s, 9H, 3 x Ac); ${ }^{13} \mathrm{C}$ NMR (DMSO-d 6 ) $\delta / \mathrm{ppm}$ : 170.0, 169.6, $169.5\left(\mathrm{CH}_{3} \mathrm{CO}\right), 155.3$ (C-4), 151.1 (C-6), 142.8 (C-7a), 120.9 (C-3a), 87.1 (C-1'), 79.5 (C-4'), 72.8 (C-2'), 70.2 (C-3'), 66.8 (C-7), 62.4 (C-5'), 20.4, 20.3, $20.3\left(\mathrm{CH}_{3} \mathrm{CO}\right)$; MS (FAB) $\mathrm{m} / z: 410\left(\mathrm{MH}^{+}, 31\right.$ $\%)$.

Anal. Calcd. for $\mathrm{C}_{16} \mathrm{H}_{19} \mathrm{~N}_{5} \mathrm{O}_{8}$ : C 46.95, H 4.68, N $17.11 \%$; found: C 47.35, H 4.63, N $16.89 \%$.

\section{6-Amino-4-chloro-1-(2,3,5-tri-O-acetyl- $\beta$-D-ribofurano- syl)-1,2,3-triazolo[4,5-c]pyridine (12)}

MeCN (50 ml, dried over $\mathrm{CaH}_{2}$ ) was directly distilled into a carefully dried three-neck flask loaded with 11 (1.5 g, 6 mmol, dried at $120{ }^{\circ} \mathrm{C}$ over $\left.\mathrm{P}_{2} \mathrm{O}_{5}\right)$ and $\mathrm{NEt}_{4} \mathrm{Cl}(1.4 \mathrm{~g}$, dried at $80{ }^{\circ} \mathrm{C}$ ) and equipped with a reflux condenser. Collidine $(0.465 \mathrm{ml}, 3.5 \mathrm{mmol})$ was then added and freshly distilled $\mathrm{POCl}_{3}(1.9 \mathrm{ml}, 21 \mathrm{mmol})$ was added dropwise. The reaction mixture was heated at $70{ }^{\circ} \mathrm{C}$ for $12 \mathrm{~min}$, the solvents were removed at a temperature below $45^{\circ} \mathrm{C}$ and the yellow residue was treated with ice (approx. $30 \mathrm{~g}$ ). After adjusting pH to 6-7 with sat. $\mathrm{NaHCO}_{3}$ at $0-5{ }^{\circ} \mathrm{C}$, the water phase was extracted with hexane $(3 \times 10 \mathrm{ml})$ and dichloromethane ( $2 \times 10 \mathrm{ml}$ ). The organic phase was dried over $\mathrm{Na}_{2} \mathrm{SO}_{4}$, the solvents were removed, and the oily residue was then applied onto a preparative column (silica gel, $20 \mathrm{~g}$ ). The foamy product $12\left(1.11 \mathrm{~g}, 70 \%\right.$, m.p. $\left.=53-55^{\circ} \mathrm{C}\right)$ was obtained after elution with benzene / EtOAc $=2: 1$.

IR (KBr) $v_{\max } / \mathrm{cm}^{-1}: 3469,3370,1741,1623,1478$, 1374, 1243, 1048, 989, 600; ${ }^{1} \mathrm{H}$ NMR $\left(\mathrm{CDCl}_{3}\right) \delta / \mathrm{ppm}: 6.37$ $(1 \mathrm{H}, \mathrm{s}, \mathrm{H}-7), 6.22\left(1 \mathrm{H}, \mathrm{d}, \mathrm{H}-1^{\prime}, J_{1^{\prime} 2^{\prime}}=3.9 \mathrm{~Hz}\right), 6.12(1 \mathrm{H}, \mathrm{dd}$, H-2'), $5.72\left(1 \mathrm{H}, \mathrm{m}, \mathrm{H}-3^{\prime}\right), 5.02\left(2 \mathrm{H}\right.$, br.s, $\left.\mathrm{NH}_{2}\right), 4.50(1 \mathrm{H}$, m, H-4'), 4.42 (1H, dd, H-5'a), 4.20 (1H, dd, H-5'b), 2.15, 2.13, $2.02\left(9 \mathrm{H}, \mathrm{s}, \mathrm{CH}_{3} \mathrm{CO}\right) ;{ }^{13} \mathrm{C}$ NMR $\left(\mathrm{CDCl}_{3}\right) \delta / \mathrm{ppm}$ : 170.54, 169.59, $169.46\left(\mathrm{CH}_{3} \underline{\mathrm{CO}}\right), 156.58(\mathrm{C}-4), 141.11$ (C-6), 138.70 (C-7a), 128.29 (C-3a), 88.21 (C-1'), 82.23 (C-7), 80.87 (C-5'), 73.28 (C-2'), $70.74\left(\mathrm{C}^{\prime} 3^{\prime}\right), 62.63$ (C-4'), 20.54, 20.45, $20.38\left(\mathrm{CH}_{3} \mathrm{CO}\right)$; MS (EI) $\mathrm{m} / z: 427\left(\mathrm{M}^{+}, 41\right.$ 
\%); HRMS Calcd. for $\mathrm{C}_{16} \mathrm{H}_{18} \mathrm{~N}_{5} \mathrm{O}_{7} \mathrm{Cl}$ : 427.090270; found: 427.089476.

Anal. Calcd. for $\mathrm{C}_{16} \mathrm{H}_{18} \mathrm{~N}_{5} \mathrm{O}_{7} \mathrm{Cl}$ : C 44.99, $\mathrm{H} 4.24, \mathrm{~N}$ $16.27 \%$; found: C 45.39, H 4.20, N $15.87 \%$.

\section{Deprotection of $\mathbf{1 2}$}

A solution of $\mathbf{1 2}$ (150 mg, $0.35 \mathrm{mmol})$ in NaOMe in $\mathrm{MeOH}$ (10 $\mathrm{ml}, 40 \mathrm{mmol}$ sol.) was stirred at room temperature for 3 h. Amberlit-IRC50 (50 mg) was added to adjust $\mathrm{pH}$ to $6.5-7$. The resin was filtered off, washed with hot water (2 $\mathrm{x} 20 \mathrm{ml}$ ) and the combined filtrates were evaporated. The resulting residue was crystallized from propanol to afford a white solid (98 mg, $93 \%$, m.p. $=155{ }^{\circ} \mathrm{C}($ dec. $)$ ).

IR (KBr) $v_{\max } / \mathrm{cm}^{-1}: 3469,3364,3298,1634,1490,1280$, 1147, 1104, 1036, 942, 802, 609; ${ }^{1} \mathrm{H}$ NMR (DMSO- $d_{6}$ ) $\delta /$ ppm: 6.63 (2H, br.s, $\left.\mathrm{NH}_{2}\right), 5.18(1 \mathrm{H}, \mathrm{s}, \mathrm{H}-7), 6.04(1 \mathrm{H}, \mathrm{d}$, $\left.\mathrm{H}-1^{\prime}, J_{1^{\prime} 2^{\prime}}=5.1 \mathrm{~Hz}\right), 4.68\left(1 \mathrm{H}, \mathrm{m}, \mathrm{H}-2^{\prime}\right), 4.22\left(1 \mathrm{H}, \mathrm{m}, \mathrm{H}-3^{\prime}\right)$, $4.00(1 \mathrm{H}, \mathrm{m}, \mathrm{H}-4)$ ') $3.56\left(2 \mathrm{H}, \mathrm{m}, \mathrm{H}-5\right.$ 'ab); ${ }^{13} \mathrm{C} \mathrm{NMR}$ (DMSO- $d_{6}$ ) $\delta /$ ppm: 157.9 (C-4), 141.0 (C-6), 140.2 (C-7a), 134.7 (C-3a), 90.6 (C-1'), 85.9 (C-5'), 81.8 (C-7), 72.8 (C-2'), 70.5 (C-3'), 61.7 (C-4'); MS (EI) m/z: $301\left(\mathrm{M}^{+}\right.$, $14 \%$ ); HRMS Calcd. for. $\mathrm{C}_{10} \mathrm{H}_{12} \mathrm{~N}_{5} \mathrm{O}_{4} \mathrm{Cl}$ : 301.058123; found: 301.057782 .

6-Amino-4-methoxy-1-( $\beta$-D-ribofuranosyl)-1,2,3-triazolo[4,5-c]pyridine (13)

A solution of 12 (150 mg, $0.47 \mathrm{mmol})$ in $\mathrm{MeOH}$ (4 ml) and $\mathrm{NaOMe}(89 \mathrm{mg}, 2.33 \mathrm{mmol})$ was heated at $120{ }^{\circ} \mathrm{C}$ in a sealed pressure tube for $25 \mathrm{~h}$, cooled and neutralized with Amberlit-12-C-50 ( $80 \mathrm{mg}$; $\mathrm{pH}=6.5-7)$. The resin was filtered off and washed with hot water $(2 \times 10 \mathrm{ml})$. The filtrate was evaporated and the residue was purified by column chromatography (silica gel, $40 \mathrm{~g}$ ) with $\mathrm{CHCl}_{3} / \mathrm{MeOH}$ $=10: 1$ mixture to yield $\mathbf{1 4}$ as yellow crystals $(60 \mathrm{mg}, 43$ $\%$, m.p. $\left.=178-182{ }^{\circ} \mathrm{C}\right)$; IR (KBr) $v_{\max } / \mathrm{cm}^{-1}: 3411,3335$, 3281, 1627, 1498, 1389, 1279, 1197, 1111, 1059; ${ }^{1} \mathrm{H}$ NMR (DMSO- $d_{6}$ ) $\delta /$ ppm: $6.18\left(2 \mathrm{H}\right.$, br.s, $\left.\mathrm{NH}_{2}\right), 6.07(1 \mathrm{H}, \mathrm{s}, \mathrm{H}-7)$, $5.93\left(1 \mathrm{H}, \mathrm{d}, \mathrm{H}-1^{\prime}\right), 4.64\left(1 \mathrm{H}, \mathrm{dd}, \mathrm{H}-2^{\prime}, J_{1^{\prime} 2^{\prime}}=4.6 \mathrm{~Hz}, J_{2^{\prime} 3^{\prime}}=\right.$ $8.4 \mathrm{~Hz}), 4.18$ (1H, m, H-3'), 4.00 (3H, s, OMe), $3.97(1 \mathrm{H}$, m, H-4'), 3.57 (1H, m, H-5'a), 3.47 (1H, dd, H-5'b, $J_{1}=$ $11.7 \mathrm{~Hz}, J_{2}=5.2 \mathrm{~Hz}$ ); ${ }^{13} \mathrm{C}$ NMR (DMSO- $\left.d_{6}\right) \delta / \mathrm{ppm}: 157.0$ (C-4), 154.5 (C-6), 142.1 (C-7a), 126.6 (C-3a), 90.1 (C-1'), 85.7 (C-4'), 75.7 (C-7), 72.6 (C-2'), 70.6 (C-3'), 61.9 (C-5'), 53.3 (OMe); MS (FAB) m/z: $298\left(\mathrm{MH}^{+}, 22 \%\right)$; HRMS Calcd. for $\mathrm{C}_{11} \mathrm{H}_{15} \mathrm{~N}_{5} \mathrm{O}_{5}$ : 298.115544; found: 298.115144 .

\section{4-Amino-6-methoxy-1-(2,3-O-isopropylidene- $\beta-D$-ribo-} furanosyl)triazolo[4,5-c]pyridine

To a solution of $\mathbf{1 3}(50 \mathrm{mg}, 0.17 \mathrm{mmol})$ in dry acetone, triethylortoformate $(110 \mu \mathrm{l}, 0.16 \mathrm{mmol})$ and $p$-toluenesulfonic acid monohydrate (30 mg, $0.03 \mathrm{mmol}$ ) were added and the resulting mixture was stirred under $\mathrm{N}_{2}$ at room temperature for $5 \mathrm{~h}$. The solution was evaporated and the residue was purified by column chromatography (silica gel, 7 g, EtOAc). Recrystallization from water afforded a white crystalline solid (40 mg, $70 \%$, m.p. $\left.=154-155{ }^{\circ} \mathrm{C}\right)$; IR $(\mathrm{KBr})$ $v_{\max } / \mathrm{cm}^{-1}: 3430,3334,3223,1644,1607,1491,1290$, $1219,1162,1027,972,866,747 ;{ }^{1} \mathrm{H}$ NMR (DMSO- $d_{6}$ ) $\delta /$ ppm: $7.32\left(2 \mathrm{H}, \mathrm{s}, \mathrm{NH}_{2}\right), 6.42\left(1 \mathrm{H}, \mathrm{d}, \mathrm{H}-1^{\prime}, J_{1^{\prime} 2^{\prime}}=2.0 \mathrm{~Hz}\right)$, $6.28(1 \mathrm{H}, \mathrm{s}, \mathrm{H}-7), 5.59$ (1H, dd, H-2'), $5.02\left(1 \mathrm{H}, \mathrm{m}, \mathrm{H}-3^{\prime}\right)$, $4.20(1 \mathrm{H}, \mathrm{m}, \mathrm{H}-4), 3.82\left(3 \mathrm{H}, \mathrm{s}, \mathrm{OCH}_{3}\right), 3.28(2 \mathrm{H}, \mathrm{m}$, $\mathrm{H}-5$ 'ab), $1.55,1.37$ (6H, s, $\left.\left(\mathrm{CH}_{3}\right)_{2} \mathrm{C}\right) ;{ }^{13} \mathrm{C}$ NMR (DMSO- $\left.d_{6}\right)$ $\delta /$ ppm: 162.95 (C-4), 150.54 (C-6), 141.19 (C-7a), 128.12 (C-3a), $\left.112.90 \quad \mathrm{CH}_{3} \mathrm{CCH}_{3}\right), 90.75$ (C-1'), 87.62 (C-4'), 83.00 (C-2'), $81.70\left(\mathrm{C}-3^{\prime}\right), 74.75$ (C-7), $61.05\left(\mathrm{C}-5^{\prime}\right), 54.04$ $\left(\mathrm{OCH}_{3}\right), 26.75,24.98\left(\mathrm{CH}_{3} \mathrm{CCH}_{3}\right)$; MS (EI) m/z: $337\left(\mathrm{M}^{+}\right.$, $10 \%$ ); HRMS Calcd for $\mathrm{C}_{14} \mathrm{H}_{19} \mathrm{~N}_{5} \mathrm{O}_{5}$ : 337.139410; found: 337.138619.

Anal. Calcd. for $\mathrm{C}_{14} \mathrm{H}_{19} \mathrm{~N}_{5} \mathrm{O}_{5}: \mathrm{C} 49.83, \mathrm{H} 5.68, \mathrm{~N}$ $20.47 \%$; found: C 49.71, H 5.60, N $20.47 \%$.

Depurination of 13: 4-Amino-6-methoxy-1H-1,2,3-triazolo[4,5-c]pyridine.

Compound 6 (100 mg, $0.34 \mathrm{mmol}$ ) was dissolved in $\mathrm{MeOH}$ (20 ml) saturated with gaseous $\mathrm{HCl}$ at $0{ }^{\circ} \mathrm{C}$, and the solution was stirred in a pressure flask at room temperature overnight. The resulting mixture was evaporated and the residue was recrystallized from $\mathrm{MeOH}$. Basic heterocycle 4-amino-6-methoxy-1H-1,2,3-triazolo[4,5-c]pyridine was isolated in $29 \%$ yield.

${ }^{1} \mathrm{H}$ NMR $\left(\mathrm{D}_{2} \mathrm{O}\right) \delta / \mathrm{ppm}: 6.22(1 \mathrm{H}, \mathrm{s}, \mathrm{H}-7), 3.82(3 \mathrm{H}, \mathrm{s}$, $\left.\mathrm{OCH}_{3}\right) ;{ }^{13} \mathrm{C}$ NMR $\left(\mathrm{D}_{2} \mathrm{O}\right) \delta /$ ppm: $155.4(\mathrm{C}-3 \mathrm{a}), 147.7$ (C-7a), 101.4 (C-7), 98.5 (C-4), 73.8 (C-6), $57.2\left(\mathrm{OCH}_{3}\right)$; MS (EI) $m / z: 165\left(\mathrm{M}^{+}, 100 \%\right)$; HRMS Calcd. for $\mathrm{C}_{6} \mathrm{H}_{7} \mathrm{~N}_{5} \mathrm{O}$ : 165.065260; found: 165.065060 .

4,6-Diamino-1-(ß-D-ribofuranosyl)-1,2,3-triazolo-[4,5-c]pyridine (15)

A suspension of $\mathbf{1 2}(150 \mathrm{mg}, 0.35 \mathrm{mmol})$ in liquid $\mathrm{NH}_{3}(30$ $\mathrm{ml})$ was heated at $120{ }^{\circ} \mathrm{C}$ in an autoclave reactor for $16 \mathrm{~h}$. After cooling, $\mathrm{NH}_{3}$ was allowed to evaporate and the residue was redissolved in $\mathrm{MeOH}$ and neutralized $(\mathrm{pH}=7)$, then purified on a preparative silica gel plate $(\mathrm{MeOH} / \mathrm{EtOAc}=$ 1:4) and recrystallized from EtOH to furnish a crystalline solid (43 mg, $43 \%$, m.p. $\left.=214-217{ }^{\circ} \mathrm{C}\right) ;{ }^{1} \mathrm{H}$ NMR (DMSO-d $\left.)_{6}\right) \delta /$ ppm: $7.62\left(2 \mathrm{H}\right.$, br.s, $\left.\mathrm{NH}_{2}\right), 7.24$ (2H, br.s, $\left.\mathrm{NH}_{2}\right), 5.87$ (1H, d, H-1'), 5.72 (1H, s, H-7), $4.64(1 \mathrm{H}, \mathrm{m}$, H-2'), 4.20 (1H, m, H-3'), 2.19 (1H, m, H-4'), 3.63 (1H, m, H-5'a), 3.47 (1H, m, H-5'b); ${ }^{13} \mathrm{C}$ NMR (DMSO- $d_{6}$ ) $\delta /$ ppm: 160.9 (C-4), 151.1 (C-6), 141.0 (C-7a), 126.5 (C-3a), 90.1 (C-1'), 85.6 (C-4'), 72.6 (C-7), 72.1 (C-2'), 70.7 (C-3'), 62.2 (C-5'); MS (EI) $\mathrm{m} / z$ : $282\left(\mathrm{M}^{+}, 4 \%\right)$; HRMS Calcd. for $\mathrm{C}_{10} \mathrm{H}_{14} \mathrm{~N}_{6} \mathrm{O}_{4}$ : 282.108650; found: 282.107653 .

\section{6-Amino-1-( $\beta$-D-ribofuranosyl)-1,2,3-triazolo[4,5-c]- pyridine (14)}

To a suspension of $\mathbf{1 2}(150 \mathrm{mg}, 0.35 \mathrm{mmol})$ in water (30 $\mathrm{ml}), \mathrm{NaOH}$ (55 mg, $1.36 \mathrm{mmol}$ ) was added and the mixture was stirred at room temperature until compound $\mathbf{1 2}$ was completely dissolved. A catalyst (50 mg, $5 \% \mathrm{Pd} / \mathrm{C}$ ) was then added and the resulting mixture was hydrogenated in a Parr hydrogenator at room temperature and 3.3 bar for 40 min. Amberlit-IRC-50 was added to the suspension, which was stirred overnight, filtered, the cake was washed twice with hot water $(10 \mathrm{ml})$ and the filtrate was evaporated. The residue was dissolved in $\mathrm{MeOH}$ and purified on preparative 
chromatographic plate in $\mathrm{MeOH} / \mathrm{EtOAc}=1: 4$ to furnish slightly yellow crystals $\left(86 \mathrm{mg}, 92 \%, \mathrm{~m} . \mathrm{p} .=182-186{ }^{\circ} \mathrm{C}\right)$; $\operatorname{IR}(\mathrm{KBr}) v_{\max } / \mathrm{cm}^{-1}: 3344,3257,1631,1564,1414,1254$, 1114,$623 ;{ }^{1} \mathrm{H}$ NMR (DMSO- $\left.d_{6}\right) \delta / \mathrm{ppm}: 8.87(1 \mathrm{H}, \mathrm{d}, \mathrm{H}-4$, $\left.J_{47}=0.98 \mathrm{~Hz}\right), 6.79\left(1 \mathrm{H}, \mathrm{d}, \mathrm{H}-7, J_{67}=0.98 \mathrm{~Hz}\right), 6.31(2 \mathrm{H}$, br.s, $\left.\mathrm{NH}_{2}\right), 6.28\left(1 \mathrm{H}, \mathrm{d}, \mathrm{H}-1^{\prime}, J_{1^{\prime} 2^{\prime}}=4.2 \mathrm{~Hz}\right), 5.01(1 \mathrm{H}, \mathrm{m}$, H-2'), 4.59 (1H, m, H-3'), $4.27\left(1 \mathrm{H}, \mathrm{m}, \mathrm{H}-4^{\prime}\right), 3.85(1 \mathrm{H}, \mathrm{m}$, H-5'a), 3.73 (1H, m, H-5'b); ${ }^{13} \mathrm{C}$ NMR (DMSO- $d_{6}$ ) $\delta / \mathrm{ppm}$ : 158.4 (C-6), 142.5 (C-4), 138.9 (C-C-7a), 138.3 (C-3a), 90.1 (C-1'), 85.8 (C-4'), 82.2 (C-C-7), 72.6 (C-2'), 70.6 (C-3'), 61.8 (C-5'); MS (EI) m/z: $267\left(\mathrm{M}^{+}, 3 \%\right)$; HRMS Calcd. for $\mathrm{C}_{10} \mathrm{H}_{13} \mathrm{~N}_{5} \mathrm{O}_{4}$ : 267.097750; found: 267.096754.

\section{4-Amino-1-( $\beta$-D-ribofuranosyl)-1,2,3-triazolo[4,5-c]- pyridine- $6(5 \mathrm{H})$-on $(2)$}

To a solution of 9 (50 mg, $0.13 \mathrm{mmol})$ in $80 \% \mathrm{EtOH}(50$ $\mathrm{ml}), \mathrm{Pd} / \mathrm{C}(30 \mathrm{mg}, 5 \%)$ was added and the suspension was hydrogenated in a Parr hydrogenator at room temperature and 3.3 bar for $3 \mathrm{~h}$. The catalyst was filtered off and washed twice with hot EtOH $(10 \mathrm{ml})$. Next, the filtrate was evaporated and the residue was recrystallized from $\mathrm{MeOH} /$ EtOAc $=2: 3$ to yield $2\left(10 \mathrm{mg}, 30 \%\right.$, m.p. $\left.=218-222{ }^{\circ} \mathrm{C}\right)$; IR (KBr) $v_{\max } / \mathrm{cm}^{-1}: 3389,3188,2924,2768,1695,1619$, 1487, 1408, 1260, 1209, 1112, 1052, 771, 563; ${ }^{1} \mathrm{H}$ NMR $\left(\mathrm{DMSO}-d_{6}\right) \delta / \mathrm{ppm}: 10.4$ (1H, br.s, NH), 7.29 (2H, br.s, $\left.\mathrm{NH}_{2}\right), 5.88\left(1 \mathrm{H}, \mathrm{d}, \mathrm{H}-1^{\prime}, J_{1^{\prime} 2^{\prime}}=5.4 \mathrm{~Hz}\right), 5.68(1 \mathrm{H}, \mathrm{s}, \mathrm{H}-3)$, 5.47 (1H, br.s, 2'-OH), 5.23 (1H, br.s, 3'-OH), $4.87(1 \mathrm{H}$, br.s, 5'-OH), 4.61 (1H, m, H-2'), 4.18 (1H, m, H-3'), 3.94 $\left(1 \mathrm{H}, \mathrm{m}, \mathrm{H}-4\right.$ ') 3.52 (2H, m, H-5'ab); ${ }^{13} \mathrm{C}$ NMR (DMSO- $d_{6}$ ) $\delta /$ ppm: 161.6 (C-6), 149.3 (C-4), 142.2 (C-3a), 115.6 (C-7a), 89.9 (C-1'), 85.5 (C-4'), 73.4 (C-7), 72.4 (C-2'), 70.4 (C-3'), 61.8 (C-5'); MS (EI) m/z: $283\left(\mathrm{M}^{+}, 13 \%\right)$; MS (FAB) $m / z$ : $284\left(\mathrm{M}^{+}+1\right)$; HRMS Calcd. for $\mathrm{C}_{10} \mathrm{H}_{13} \mathrm{~N}_{5} \mathrm{O}_{5}$ : 283.091669; found: 283.091890 .

\section{CONCLUSIONS}

In conclusion, $\mathbf{5}$ can be used in a novel base-catalyzed ring closure for the synthesis of a variety of 2-alkoxy-8-aza-3-deazaadenosines, namely new 8-azaspongosine analogues. ${ }^{12}$ The proper choice of alkoxides (e.g., sodium benzylate) enables easy deprotection of $\mathbf{9}$ to the target 8-aza-3-deazaisoguanosine 2. Further studies of its self-assembling properties are in progress.

Acknowledgements. - The authors thank Dr. Bogdan Kralj of the Mass Spectrometry Centre at Jozef Stefan Institute (Ljubljana) and Janez Plavec of the Slovenian National NMR Centre. This investigation was supported by the Ministry of Science and Technology of Slovenia (P-0515) and COST-D8 Programme.

\section{REFERENCES}

1. (a) C. Switzer, S. E. Moroney, and S. A. Benner, Biochemistry 32 (1993) 10489-10496; (b) C. Roberts, R. Bandaru, and C. Switzer, J. Am. Chem. Soc. 119 (1997) 4640-4649.
2. T. Horn, C. A. Chang, and M. L. Collins, Nucleosides\&Nucleotides 14 (1995) 1023-1026.

3. E. T. Kool, J. C. Morales, and K. M. Guckian, Angew. Chem., Int. Ed. Engl. 39 (2000) 990-1009.

4. T. Lan and W. McLaughlin, J. Am. Chem. Soc. 122 (2000) 6512-6513.

5. (a) W. Guschlbauer, J. F. Chantot, and P. Thiele, J. Biomol. Str. Dyn. 8 (1990) 491-511; (b) G. Gottarelli, S. Masiero, and G. Spada, Chem. Commun. (1995) 2555-2557.

6. (a) J. T. Davis, S. K. Tirumala, and A. L. Marlow, J. Am. Chem. Soc. 119 (1997) 5271-5272; (b) M. Cai, A. L. Marlow, J. C. Fettinger, D. Fabris, T. J. Haverlock, B. A. Moyer, and J. T. Davis, Angew. Chem., Int. Ed. Engl. 39 (2000) 1283-1285; (c) J. T. Davis, S. Tirumala, J. R. Jenssen, D. Radler, and E. J. Fabris, J. Org. Chem. 60 (1995) 4167-4176; (d) S. Tirumala and J. T. Davis, J. Am. Chem. Soc. 119 (1997) 2769-2776.

7. X. Shi, J. C. Fettinger, M. Cai, and J. T. Davis, Angew. Chem., Int. Ed. Engl. 39 (2000) 3124-3127.

8. J. L. Sessler, M. Sathiosatham, K. Doerr, C. H. Lynch, and K. A. Abboud, Angew. Chem., Int. Ed. Engl. 39 (2000) 1300-1303.

9. D. Sen and W. Gilbert, Nature 344 (1990) 410-414.

10. A. Mazunder, N. Neamati, J. O. Ojwang, S. Sunder, R. F. Rando, and Y. Pommier, Biochemistry 35 (1996) 1376213771.

11. (a) F. Seela, C. Wei, A. Malenewski, and E. Feiling, $N u$ cleosides\&Nucleotides 17 (1998) 2045-2052; (b) F. Seela and C. Wei, Chem. Commun. (1997) 1869-1870; (c) F. Seela and C. Wei, Helv. Chim. Acta 80 (1997) 73-85.

12. (a) F. A. Fuhrman and G. J. Fuhrman, Comp. Biochem. Physiol. 72C (1982) 203-210; (b) M. Ueeda, R. D. Thompson, L. H. Arroyo, and A. Olsson, J. Med. Chem. 34 (1991) 1334-1339.

13. (a) A. Štimac, I. Leban, and J. Kobe, Synlett (1999) 1069-1073; (b) A. Štimac, L. B. Townsend, and J. Kobe, Nucleosides\&Nucleotides 10 (1991) 727-728.

14. (a) R. A. Earl and L. B. Townsend, Can. J. Chem. 58 (1980), 2550-2561; (b) R. B. Meyer, G. P. Revankar, D. Cook, K. W. Ehler, M. P. Schweizer, and R. K. Robins, J. Het. Chem. 17 (1980) 159-169; (c) D. P. Cook and B. Rousseau, J. Am. Chem. Soc. 97 (1975) 2916-2917; (d) G. P. Revankar, P. K. Gupta, A. D. Adams, N. K. Dalley, P. A. McKernan, D. P. Cook, P. G. Canonico, and R. K. Robins, J. Med. Chem. 27 (1984) 1389-1396; (e) D. A. Berry, R. B. Gilbertsen, and D. P. Cook, ibid. 29 (1986) 2034-2037; (f) N. Miakawa, Y. Sasabuchi, A. Kiyosue, N. Kojima, and A. Matsuda, Chem. Pharm. Bull. 44 (1996) 288-295; (g) N. Minakawa and A. Matsuda, Tetrahedron 49 (1993) 557-570; (h) N. Minakawa and A. Matsuda, Tetrahedron Lett. 34 (1993) 661-664; (i) H. Tanaka and T. Ueda, J. Het. Chem. 16 (1979) 411-412; (j) H. Tanaka, M. Hirayama, M. Suzuki, T. Miyasaka, A. Matsuda, and T. Ueda, Tetrahedron 42 (1986) 1971-1980.

15. D. P. Cook and R. K. Robins, J. Org. Chem. 43 (1978) 289-293; (b) B. L. Booth, F. A. T. Costa, Z. Mahmoud, R. G. Pritchard, and M. F. Proenca, J. Chem. Soc., Perkin Trans. 1 (1999) 1853-1858.

16. F. Johnson and W. A. Nasutavicus, J. Org. Chem. 27 (1962) 3953-3958.

17. (a) R. Tan and A. Taurins, Tetrahedron Lett. (1965) 2737; (b) A. Taurins and R. Tan Li, Can. J. Chem. 52 (1974) 843-848; (c) F. Alhaique, F. M. Riccieri, and E. Santucci, Tetrahedron Lett. 11 (1975) 173-174. 
18. R. K. Robins, J. K. Horner, C. V. Greco, C. W. Noell, and C. G. Beames, J. Org. Chem. 28 (1963) 3041-3046.

19. R. J. Rousseau, J. A. May, R. K. Robins, and L. B. Townsend, J. Het. Chem. 11 (1974) 233-235.

20. M. Ueeda, R. D. Thompson, L. H. Arroyo, and R. A. Olsson, J. Med. Chem. 34 (1991) 1340-1344.
21. M. J. Wu, Li-J. Chang, Li-M. Wei, and C. F. Lin, Tetrahedron 55 (1999) 13193-13200; (b) S. R. Baker, L. Crombi, R. V. Dove, and D. A. Slack, J. Chem. Soc., Perkin Trans. 1 (1979) 677-685.

22. J. L. Imbach, J. L. Barascut, B. Kam, and C. Tapiero, Tetrahedron Lett. (1974) 129-130.

\section{SAŽETAK}

\section{Priprava 8-aza-3-deazaizoguanozina s novim zatvaranjem prstena pomoću natrijeva alkoksida}

\section{Marjan Ješelnik, Suzana Jakša i Jože Kobe}

U članku je opisano izravno zatvaranje prstena 4-ciano-5-cianometil-1-( $\beta$-D-ribofuranizil)-1,2,3-triazolo[4,5-c]piridina u bazičnim uvjetima u 4-amino-5-alkoksi-1-( $\beta$-D-ribofuranizil)-1,2,3-triazolo[4,5-c]piridin, koji se lako transformira u 8-aza-3-deazaizoguanozin. 